\title{
Arkadia protein expression is reduced in the liver during the progression of hepatic fibrosis
}

\author{
FEI HOU ${ }^{1,2^{*}}$, RUIXIA LIU ${ }^{1,3^{*}}$, XIAOYA LIU ${ }^{1}$, LIJIAN CUI $^{1}$, XIAOZHENG YU ${ }^{1}$, \\ YAN WEN $^{1}$, HUIGUO DING ${ }^{4}$ and CHENGHONG YIN ${ }^{3}$ \\ ${ }^{1}$ Department of Infection, Beijing Friendship Hospital, Capital Medical University, Beijing 100050; \\ ${ }^{2}$ Department of Intensive Care Unit, Beijing Ditan Hospital, Capital Medical University, Beijing 100015; \\ ${ }^{3}$ Department of Internal Medicine, Beijing Obstetrics and Gynecology Hospital, Capital Medical University, \\ Beijing 100026; ${ }^{4}$ Gastroenterology and Hepatology Department, Beijing Youan Hospital, \\ Capital Medical University, Beijing 100069, P.R. China
}

Received June 16, 2016; Accepted November 30, 2017

DOI: 10.3892/ijmm.2017.3340

\begin{abstract}
Arkadia is able to degrade key signaling molecules in the transforming growth factor (TGF)- $\beta 1$ signaling pathway; however, the expression of Arkadia in the liver during development and progression of TGF- $\beta 1 /$ Smad signaling-regulated hepatic fibrosis remains to be elucidated. The present study aimed to examine Arkadia expression in the livers of two rat models of hepatic fibrosis induced by bile duct ligation and carbon tetrachloride intoxication, and in human liver samples from patients with hepatic fibrosis. Expression was analyzed by quantitative polymerase chain reaction, immunohistochemistry and western blot analysis. The results indicated that Arkadia was predominantly expressed in the cytoplasm of cholangiocytes and hepatocytes. The protein expression levels of Arkadia were significantly decreased in fibrotic livers, whereas the mRNA expression levels of Arkadia were significantly increased in fibrotic livers compared with in nonfibrotic livers. In conclusion, these data indicated that Arkadia may regulate the pathogenesis and progression of hepatic fibrosis.
\end{abstract}

\section{Introduction}

Hepatic fibrosis is a common pathophysiological outcome of various chronic hepatic diseases, which may be caused by viral

Correspondence to: Professor Chenghong Yin, Department of Internal Medicine, Beijing Obstetrics and Gynecology Hospital, Capital Medical University, 251 Yaojiayuan Road, Chaoyang, Beijing 100026, P.R. China

E-mail: modscn@126.com

Dr Huiguo Ding, Gastroenterology and Hepatology Department, Beijing Youan Hospital, Capital Medical University, 8 You'an Men Wai Xi Tou Tiao Road, Fengtai, Beijing 100069, P.R. China

E-mail: dinghuiguo@ccmu.edu.cn

*Contributed equally

Key words: Arkadia, hepatic fibrosis, E3 ubiquitin ligase, transforming growth factor- $\beta 1$ signaling pathway, post-translational modification infection, alcohol abuse, drug or chemical toxicity, hepatic autoimmunity, biliary obstruction, etc. During the process of hepatic injury, injured hepatocytes degenerate, and stimulate hepatocyte regeneration and liver inflammation (1). Chronic hepatocyte injury and degeneration is able to promote the deposition of extracellular matrix components, including collagens, glycoproteins, mucopolysaccharide and proteoglycan, thus leading to liver fibrosis (2). Without optimal control, hepatic fibrosis can progress to cirrhosis, hepatocellular carcinoma and liver failure (3). Cirrhosis and hepatocellular carcinoma are major causes of morbidity and mortality worldwide (4); however, the molecular mechanisms underlying hepatic fibrosis remain unclear.

Post-translational modification by protein ubiquitination can degrade misfolded and unwanted proteins through proteasomes. This process affects various signaling processes, including cell signaling, DNA repair, protein trafficking, chromatin modifications, cell cycle progression and cell death (5). Arkadia is a RING-type E3 ubiquitin ligase, also known as ring finger protein 11 , which can catalyze degradation of key signaling molecules in the transforming growth factor (TGF)- $\beta 1$ signaling pathway $(6,7)$. TGF- $\beta 1$ is a profibrotic factor that activates Smad signaling and is closely associated with the development and progression of hepatic fibrosis (8-10). Previous studies have reported that Arkadia can degrade Smad6, Smad7, c-Ski and SnoN, which are negative regulators of the TGF- $\beta 1$ signaling (6,11-14). However, little is currently known regarding the expression levels of Arkadia during the process of hepatic fibrosis, and the location of Arkadia expression in the liver.

The present study employed two rat models of hepatic fibrosis in order to characterize the expression levels of Arkadia in fibrotic livers from rats, as well as liver samples from patients with various stages of hepatic fibrosis. The results indicated that Arkadia was predominantly expressed in the cytoplasm, whereas expression was reduced in the nucleus of hepatocytes and cholangiocytes in liver samples from rats with hepatic fibrosis. The relative mRNA expression levels of Arkadia were increased in the livers of rats with hepatic fibrosis, whereas the protein expression levels of Arkadia were gradually reduced in the livers of rats hepatic fibrosis and in human liver samples from patients with hepatic fibrosis. 


\section{Materials and methods}

Human liver samples. Human liver tissues used in the present study were remnants from biopsies obtained for routine clinical purposes or were cirrhotic liver samples acquired during hepatectomy and were collected between September 2014 and July 2015. Each liver biopsy sample was processed for routine histology. Informed consent was obtained from each patient prior to participation in the present study. The experimental protocol was approved by the Ethics Committee of Beijing Friendship Hospital, Capital Medical University (Beijing, China).

Animals. Male Wistar rats (weight, 180-220 g) were obtained from the Laboratory Animal Center of Chinese Academy of Medical Sciences (Beijing, China). The rats were housed in a specific pathogen-free facility with controlled temperature $\left(25 \pm 1^{\circ} \mathrm{C}\right)$ and humidity $(55 \pm 5 \%)$, under a 12 -h light/dark cycle. The rats were allowed free access to rat chow and water. All animals received humane care according to the criteria outlined in the 'Guide for the Care and Use of Laboratory Animals' prepared by the National Academy of Sciences and published by the National Institutes of Health (NIH publication no. 86-23). The animal study was approved by the Ethics Committee of Beijing Friendship Hospital, Capital Medical University.

\section{Animal models of hepatic fibrosis.}

Carbon tetrachloride $\left(\mathrm{CCl}_{4}\right)$ intoxication. The rats were randomly assigned to five groups ( $\mathrm{n}=6$ rats/group). The control group was injected intraperitoneally (i.p.) with olive oil ( $0.2 \mathrm{ml} / 100 \mathrm{~g}$ body weight) twice per week for 8 consecutive weeks. The hepatic fibrosis groups were injected with $50 \%$ $\mathrm{CCl}_{4}$ in olive oil (i.p; Sinopharm Chemical Reagent Co., Ltd., Shanghai, China) at a dose of $0.15 \mathrm{ml} / 100 \mathrm{~g}$ body weight twice per week for 2, 4, 6 or 8 weeks. After the final injection, rats were fasted overnight and anesthetized with pentobarbital sodium (40 mg/kg). The rats were then sacrificed. Subsequently, blood and liver samples were collected for further analysis.

Bile duct ligation $(B D L)$. The rats were randomly divided into five groups ( $\mathrm{n}=6$ rats/group), including the sham operation group, and BDL 7, 14, 28 and 42 day groups. The rats were anesthetized with pentobarbital sodium, and the BDL and sham operations were performed, as previously described $(15,16)$. Briefly, a 2-cm midline laparotomy was performed, after which the common bile duct was identified and ligated twice with 6.0 silk sutures (17). The first ligature was made under the junction of the hepatic ducts and the second ligature was located above the entrance to the pancreatic ducts. The rats in the sham operation group were subjected to abdominal incision, and the common bile duct was identified but not ligated. After the abdomen was sutured, the animals were recovered on a heating pad. After $24 \mathrm{~h}$, the rats were allowed free access to food and water. The rats in the sham operation group were sacrificed 42 days post-surgery. The rats in the experimental groups were sacrificed at 7, 14, 28 or 42 days post-surgery. Liver and blood samples were obtained for further analysis.

Detection of serum biochemical indicators. Individual serum samples were prepared by centrifugation at $1,811 \mathrm{x} g$ for $5 \mathrm{~min}$ at $4^{\circ} \mathrm{C}$, and the levels of serum aspartate transaminase (AST), alanine transaminase (ALT), direct bilirubin (DBIL) and total bilirubin (TBIL) were analyzed using an automatic biochemistry analyzer (Abbott Laboratories, Chicago, IL, USA), according to the manufacturer's protocol.

Histopathological examination. The liver tissues were fixed with $4 \%$ paraformaldehyde for $24 \mathrm{~h}$ at room temperature, and embedded in paraffin. Subsequently, liver tissue sections $(4 \mu \mathrm{m})$ were stained with hematoxylin and eosin (H\&E) or Masson's trichrome. Finally, the sections were dehydrated by increasing concentrations of ethanol and xylene. In Masson's staining, the sections were dewaxed and rehydrated by $\mathrm{H}_{2} \mathrm{O}$ and distilled water, and then stained by Harris's hematoxylin dye for $1 \mathrm{~min}$. After washed with $\mathrm{H}_{2} \mathrm{O}$, the sections were stained with Masson's acid magenta dye for $10 \mathrm{~min}$, differentiated by $1 \%$ phosphomolybdic acid aqueous solution for $5 \mathrm{~min}$ and washed with Glacial acetic acid for a few seconds. Images of the sections were captured under a light microscope (DM6000B; Leica Microsystems GmbH, Mannheim, Germany).

Immunohistochemistry. The paraffin-embedded liver sections $(4 \mu \mathrm{m})$ were rehydrated, and treated with $3 \% \mathrm{H}_{2} \mathrm{O}_{2}$ in methanol to inactivate endogenous peroxidase. The sections were then incubated with rabbit anti-Arkadia (sc-367716; Santa Cruz Biotechnology, Inc., Dallas, TX, USA) overnight at $4^{\circ} \mathrm{C}$. After washing, the bound antibodies were detected using horseradish peroxidase (HRP)-conjugated goat anti-rabbit immunoglobulin (Ig)G (PV-9001; OriGene Technologies, Inc., Beijing, China) at room temperature for $30 \mathrm{~min}$ and visualized using diaminobenzidine (DAB; ZLI-9017; OriGene Technologies, Inc.), followed by counterstaining with hematoxylin. The images of the different groups were captured using a microscope (Leica DM6000B; Leica Microsystems GmbH).

Reverse transcription-quantitative polymerase chain reaction $(R T-q P C R)$. Total RNA was extracted from liver tissues using TRIzol reagent (Invitrogen; Thermo Fisher Scientific, Inc., Waltham, MA, USA) and was reverse transcribed $\left(42^{\circ} \mathrm{C}\right.$ for $15 \mathrm{~min}, 95^{\circ} \mathrm{C}$ for $5 \mathrm{~min}, 4^{\circ} \mathrm{C}$ for $5 \mathrm{~min}$, only 1 cycle) into cDNA using $3 \mu \mathrm{l}$ 10X RT buffer, $4 \mu \mathrm{l} \mathrm{MgCl}_{2}, 0.5 \mu \mathrm{l}$ recombinant RNase inhibitor, $2 \mu \mathrm{l}$ dNTP mixture, $1 \mu \mathrm{l}$ Oligo dT-Adaptor primer, 0.6 $\mu$ l AMV reverse transcriptase (Promega Corp., Madison, WI, USA) and $1 \mu \mathrm{g}$ RNA sample. The relative mRNA expression levels of Arkadia in individual samples were determined by qPCR using the SYBR-Green Master Mix (Applied Biosystems; Thermo Fisher Scientific, Inc.) and specific primers in an ABI 7500 sequence detection system (Applied Biosystems; Thermo Fisher Scientific, Inc.). The thermocycling conditions were as follows: denaturation at $95^{\circ} \mathrm{C}$ for $10 \mathrm{~min}, 40$ cycles at $95^{\circ} \mathrm{C}$ for $15 \mathrm{sec}, 64^{\circ} \mathrm{C}$ for $30 \mathrm{sec}$, $95^{\circ} \mathrm{C}$ for $15 \mathrm{sec}, 60^{\circ} \mathrm{C}$ for $1 \mathrm{~min}$, and $95^{\circ} \mathrm{C}$ for $15 \mathrm{sec}$, and a final extension step at $60^{\circ} \mathrm{C}$ for $15 \mathrm{sec}$. The primer sequences were asfollows:RatArkadia,forward5'-TCATATTCATGTGCCTCA AACCA-3', reverse 5'-CCCAGTTCCCAGGCAGTTC-3'; and GAPDH, forward 5'-ACGCATTTGGTCGTATTGGG-3' and reverse 5'-TGATTTTGGAGGGATCTCGC-3'. Data were analyzed by $2^{-\Delta \Delta \mathrm{Cq}}(18)$.

Western blot analysis. Individual liver tissue samples were homogenized in radioimmunoprecipitation assay lysis buffer 


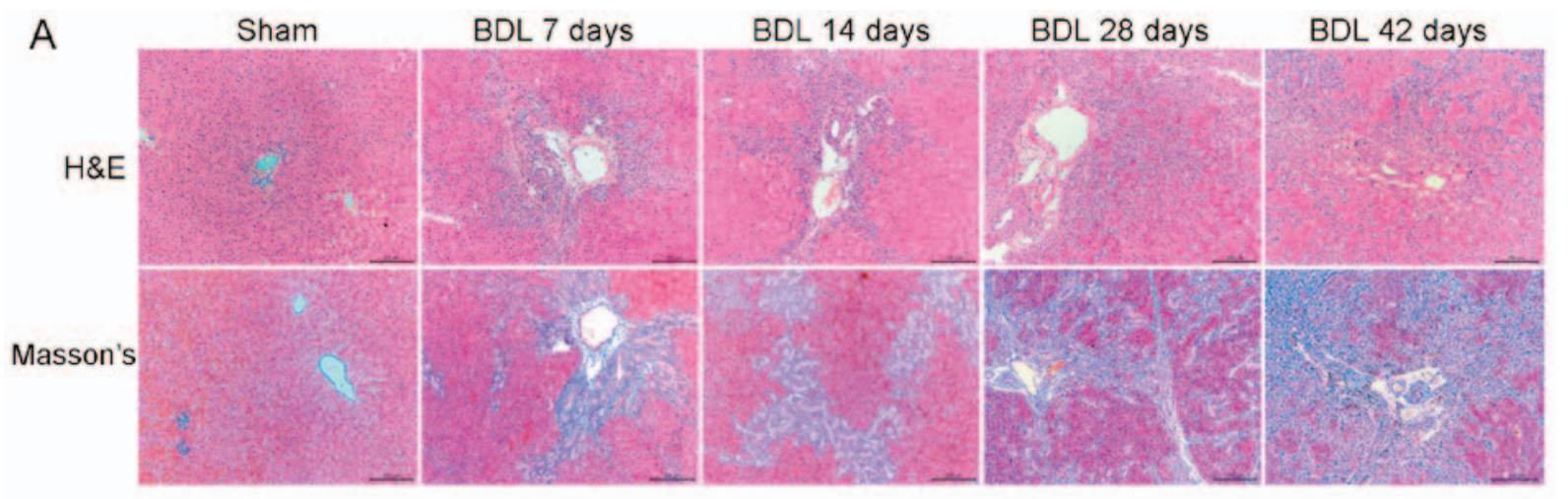

B

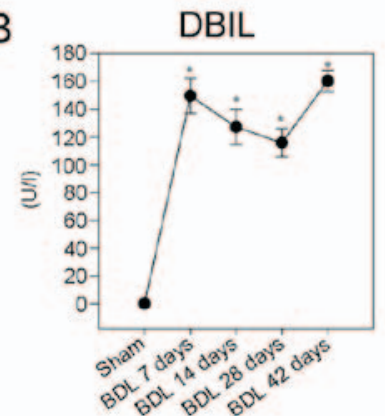

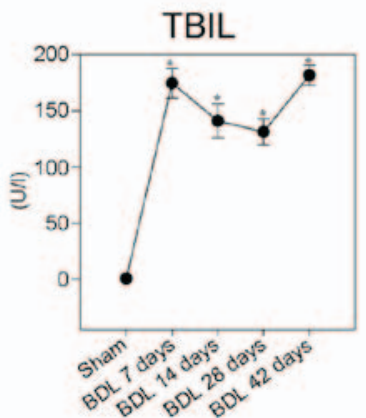
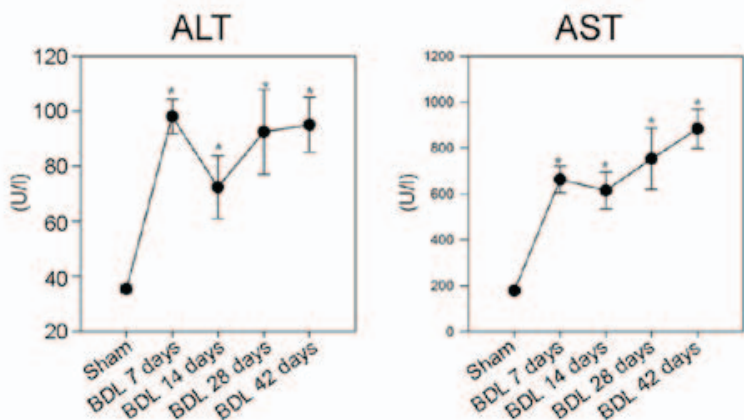

Figure 1. BDL-induced hepatic fibrosis in rats. Following BDL, rats were sacrificed at various time points post-surgery and liver sections underwent H\&E and Masson staining. The serum levels of DBIL, TBIL, ALT and AST were determined in individual rats. (A) Histological examination of the liver sections (magnification, x100). Representative images are presented. (B) Effects of BDL on serum biochemical indicators. Data are presented as the means \pm standard deviation of individual groups ( $\mathrm{n}=6 /$ group). $\mathrm{P}<0.05$ vs. the sham operation group. ALT, alanine transaminase; AST, aspartate transaminase; $\mathrm{BDL}$, bile duct ligation; DBIL, direct bilirubin; H\&E, hematoxylin and eosin; TBIL, total bilirubin.

containing a protease inhibitor cocktail (Roche Diagnostics, Indianapolis, IN, USA). After centrifugation at $12,000 \mathrm{x} \mathrm{g}$ for 15 min at $4^{\circ} \mathrm{C}$, protein concentrations were measured using the bicinchoninic acid protein assay kit (Pierce; Thermo Fisher Scientific, Inc.). Individual tissue lysates (30 $\mu \mathrm{g} / \mathrm{lane})$ were separated by $8-12 \%$ SDS-PAGE, and were transferred to polyvinylidene fluoride membranes (EMD Millipore, Billerica, MA, USA). After blocking with 5\% fat-free dry milk in Tris-buffered saline-0.05\% Tween (TBST) for $2 \mathrm{~h}$ at room temperature, the membranes were incubated with rabbit anti-Arkadia (1:500; sc-367716; Santa Cruz Biotechnology,Inc.), rabbit anti-GAPDH (1:1,000; ab181602; Abcam, Cambridge, UK), rabbit anti- $\beta$-actin antibody (1:1,000; ab8227; Abcam) at $4^{\circ} \mathrm{C}$ overnight. Subsequently, the membranes were washed three times with TBST and incubated with HRP-conjugated goat anti-rabbit IgG (PV-9001; Zhongshan Goldenbridge Biotechnology Co., Ltd., Beijing, China) for $30 \mathrm{~min}$ at room temperature. The blots were visualized using an enhanced chemiluminescence kit (EMD Millipore). The band density of target proteins was quantified and normalized to that of GAPDH using Image Lab version 5.1 software (Bio-Rad Corporation, Hercules, CA, USA).

Statistical analysis. Data are representative images or are presented as the means \pm standard deviation, unless otherwise specified. These experiments were repeated for 6 times. All statistical analyses were conducted using SPSS 20.0 software (IBM Corporation, Armonk, NY, USA). The difference between two groups was analyzed by Student's t-test. $\mathrm{P}<0.05$ was considered to indicate a statistically significant difference.

\section{Results}

Effects of BDL on liver pathology and function in rats. To evaluate the effects of BDL on liver pathology and collagen fiber deposition in rats, the rats were subjected to sham or BDL surgery and were then sacrificed at various time points post-surgery. Pathological alterations in the liver tissues were analyzed by H\&E and Masson staining. As shown in Fig. 1A, the sham group displayed normal lobular architecture with central veins and radiating hepatic cords. Conversely, small bile duct dilation, portal inflammation and hepatocyte degeneration, as well as inflammatory infiltration, were accompanied by obvious fibrosis in the livers of rats in the BDL 7 day group. Small bile duct dilation around the portal area, hepatocyte degeneration and inflammatory infiltration, as well as collagenous fibers were more obvious in the livers of rats in the BDL 14 day group. In the BDL 28 and 42 day groups, cholestasis became more severe, epithelial cells were detected in the interlobular bile ducts, and diffused collagenous fibers and capillaries extended into the hepatic lobular regions, thus forming membrane-like structures in the liver lobules; these are hallmarks of hepatic fibrosis. Masson staining revealed that the liver sections of the sham group exhibited normal distribution of collagen fibers (stained blue) around the central veins and portal tracts, whereas the number of collagenous fibers was gradually increased after BDL. In the BDL 28 and 42 day groups, marked collagen fibers were deposited around the central vein and small bile ducts, which formed membrane-like structures in the hepatic lobules, a characteristic of pseudolobules. 


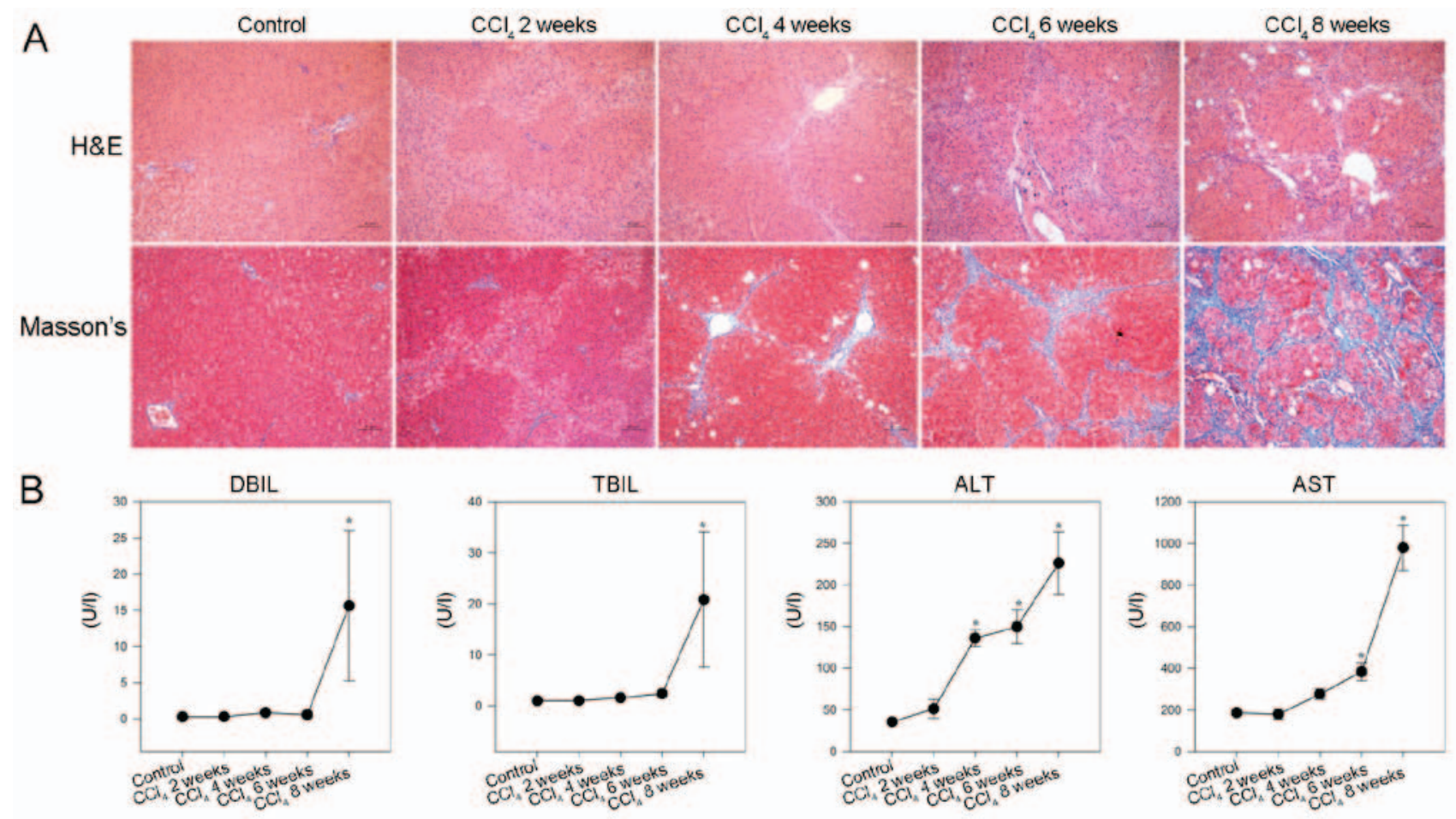

Figure 2. $\mathrm{CCl}_{4}$-induced hepatic fibrosis in rats. Following $\mathrm{CCl}_{4}$ injection, rats were sacrificed at the indicated time points and liver sections underwent H\&E and Masson staining. The serum levels of DBIL, TBIL, ALT and AST were detected in individual rats. (A) Histological examination of liver sections (magnification, x100). Representative images are presented. (B) Effects of $\mathrm{CCl}_{4}$ administration on serum biochemical indicators. Data are presented as the means \pm standard deviation of individual groups ( $\mathrm{n}=6 /$ group). ${ }^{*} \mathrm{P}<0.05$ vs. the control group. ALT, alanine transaminase; $\mathrm{AST}$, aspartate transaminase; $\mathrm{CCl} \mathrm{H}_{4}$ carbon tetrachloride; DBIL, direct bilirubin; H\&E, hematoxylin and eosin; TBIL, total bilirubin.

Further analyses indicated that the BDL groups of rats had significantly higher levels of DBIL, TBIL and ALT, and gradually increased levels of AST, compared with in the sham group of rats $(\mathrm{P}<0.05$; Fig. 1B). These results suggested that BDL may impair liver function, thus gradually resulting in liver fibrosis in rats.

Effects of $\mathrm{CCl}_{4}$ on liver pathology and function in rats. To provide additional evidence, a $\mathrm{CCl}_{4}$ model of liver fibrosis was generated in rats. As shown in Fig. 2A, hepatocyte swelling and steatosis, spotty necrosis in the hepatic lobule and inflammatory infiltrates around the portal duct areas were detected in the livers of rats in the $\mathrm{CCl}_{4} 2$ week group. Furthermore, increased inflammatory infiltrates and fibrocytes surrounding the hepatic lobules, together with hepatocyte degeneration and focal necrosis were observed in the livers of rats in the $\mathrm{CCl}_{4} 4$ week group. In addition, disarranged fibrous septa and pseudolobules were detected in the hepatic lobules of rats in the $\mathrm{CCl}_{4} 6$ and 8 week groups. Masson staining revealed that while collagen fibers were detected around the portal tracts and central veins in the livers of control rats, increased numbers of collagenous fibers were observed in the livers of $\mathrm{CCl}_{4}$-injected rats. In addition, collagenous fibers formed membrane-like structures in the hepatic lobules of rats in the $\mathrm{CCl}_{4} 8$ week group.

Consequently, significantly increased levels of serum DBIL and TBIL, as well as gradually elevated ALT and AST, were detected in the $\mathrm{CCl}_{4}$-injected rats compared with in the control rats $(\mathrm{P}<0.05$; Fig. $2 \mathrm{~B})$. These results indicated that injection with $\mathrm{CCl}_{4}$ impaired liver function and induced hepatic fibrosis in rats.
Expression of Arkadia in rats with BDL-induced hepatic fibrosis. To evaluate Arkadia expression in rats with BDL-induced hepatic fibrosis, the expression levels of Arkadia were detected in fibrotic and normal liver tissues by immunohistochemical staining. The results demonstrated that Arkadia was predominantly expressed in the cytoplasm, and was weakly expressed in the nucleus of hepatocytes and cholangiocytes (Fig. 3A). There were no notable differences between sham and BDL rat Arkadia immunohistochemistry results. Using qPCR, the mRNA expression levels of Arkadia were revealed to be significantly higher in the livers of the BDL 7 day group compared with in the control group $(\mathrm{P}<0.01)$, after which they gradually decreased; the expression levels in the livers of the BDL 28 day group were similar to those in the control group (Fig. 3B). However, the mRNA expression levels of Arkadia were significantly elevated in the liver samples of the BDL 42 day group compared with in the control group $(\mathrm{P}<0.01)$. Western blot analysis indicated that increased levels of Arkadia expression were detected in the livers of the control rats, whereas the protein expression levels of Arkadia were gradually reduced in the fibrotic livers of rats $(\mathrm{P}<0.01$; Fig. $3 \mathrm{C}$ and $\mathrm{D})$. These results indicated that the levels of Arkadia mRNA transcripts were increased and the protein expression levels of Arkadia were decreased in hepatocytes and cholangiocytes from the fibrotic livers of rats.

Expression of Arkadia in rats with $\mathrm{CCl}_{4}$-induced hepatic fibrosis. The present study used immunohistochemistry to investigate the expression of Arkadia in $\mathrm{CCl}_{4}$-induced hepatic fibrosis. Strong Arkadia staining was detected in the cytoplasm, whereas weak staining was detected in the nucleus of hepato- 

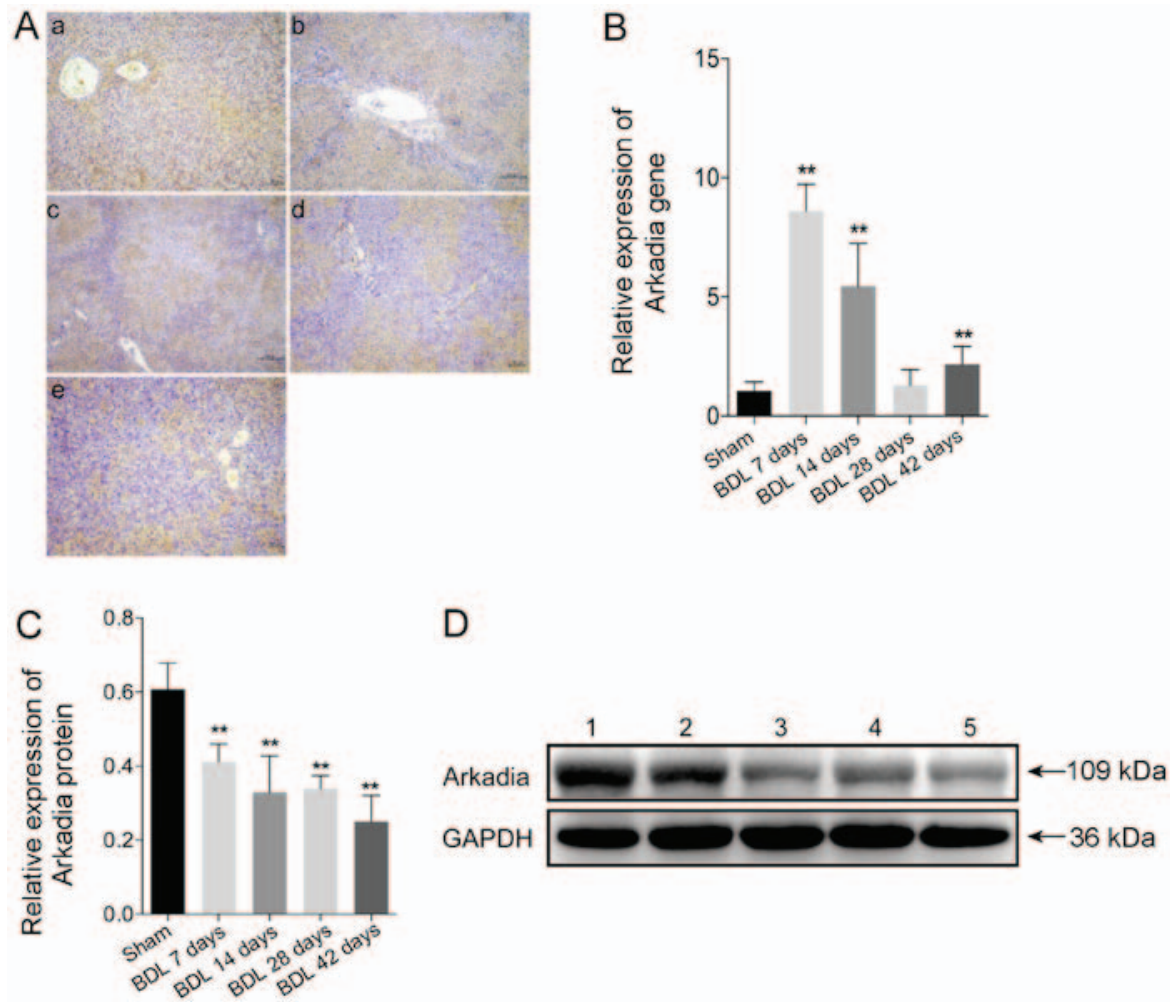

Figure 3. Arkadia expression in rats with BDL-induced hepatic fibrosis. Following sacrifice, the expression levels of Arkadia were detected in the liver samples using RT-qPCR, western blot analysis and immunohistochemistry. Data are presented as representative images or are expressed as the means \pm standard deviation of individual groups ( $\mathrm{n}=6 /$ group). (A) Immunohistochemical staining of Arkadia expression in liver sections (original magnification, x100). (a) Sham; (b) BDL 7 days; (c) BDL 14 days; (d) BDL 28 days and (e) BDL 42 days. (B) RT-qPCR analysis of Arkadia mRNA transcript levels in the liver samples. (C) Relative protein expression levels of Arkadia. (D) Western blot analysis of Arkadia protein. Lane 1, Sham; lane 2, BDL 7 days; lane 3, BDL 14 days; lane 4, BDL 28 days and lane 5, BDL 42 days. ${ }^{* *} \mathrm{P}<0.01$ vs. the sham group. BDL, bile duct ligation; RT-qPCR, reverse transcription-quantitative polymerase chain reaction.
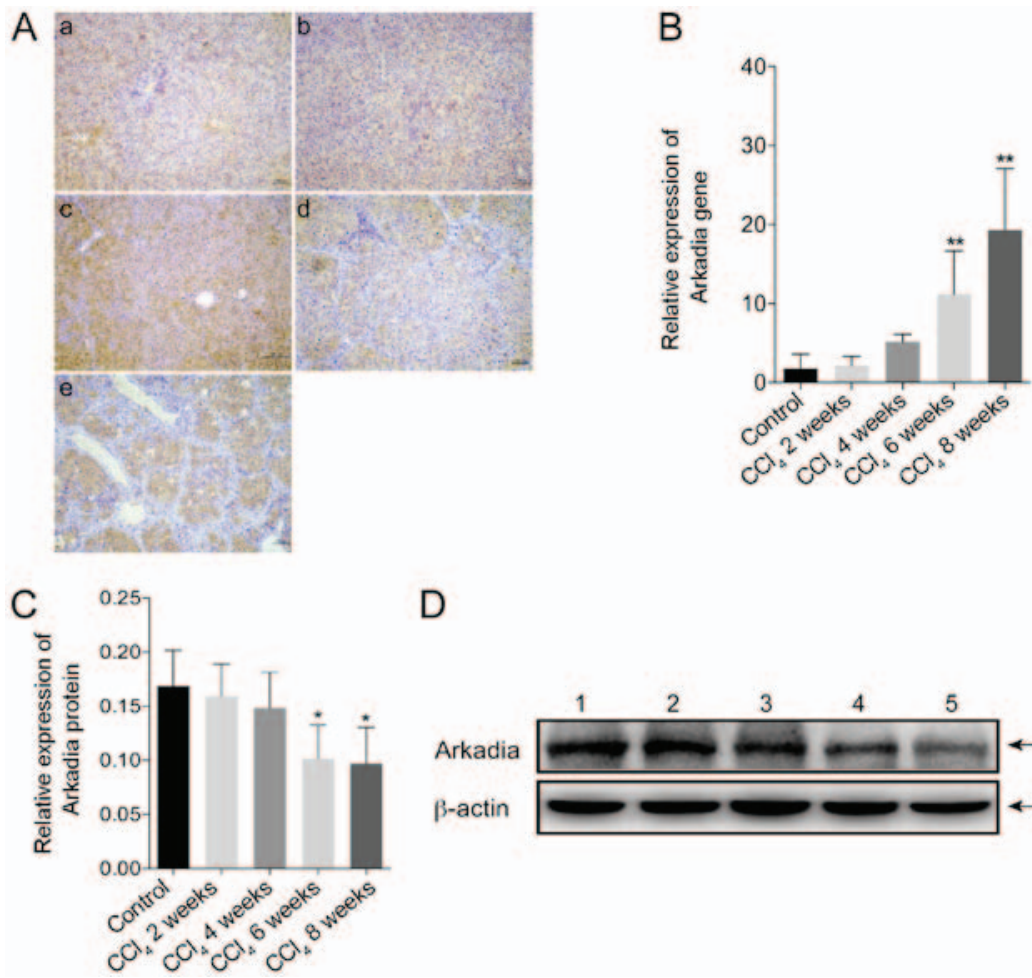

D

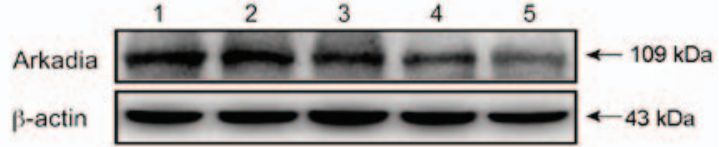

Figure 4. Arkadia expression in rats with $\mathrm{CCl}_{4}$-induced hepatic fibrosis. Following sacrifice, the expression levels of Arkadia in the liver samples were determined by RT-qPCR, western blot analysis and immunohistochemistry. Results are presented as representative images or are expressed as the means \pm standard deviation of individual groups ( $\mathrm{n}=6$ /group). (A) Immunohistochemical staining of Arkadia expression in the liver sections (original magnification, $\mathrm{x} 100$ ). (a) control; (b) $\mathrm{CCl}_{4}$ 2 weeks; (c) $\mathrm{CCl}_{4} 4$ weeks; (d) $\mathrm{CCl}_{4} 6$ weeks and (e) $\mathrm{CCl}_{4} 8$ weeks. (B) RT-qPCR analysis of Arkadia mRNA transcript levels in the liver samples. (C) Relative protein expression levels of Arkadia. (D) Western blot analysis of Arkadia protein. Lane 1, control; lane 2, $\mathrm{CCl}_{4} 2$ weeks; lane 3, $\mathrm{CCl}_{4} 4$ weeks; lane 4, $\mathrm{CCl}_{4} 6$ weeks and lane $5, \mathrm{CCl}_{4} 8$ weeks. ${ }^{*} \mathrm{P}<0.05,{ }^{* * *} \mathrm{P}<0.01$ vs. the control group. $\mathrm{CCl}_{4}$, carbon tetrachloride; RT-qPCR, reverse transcription-quantitative polymerase chain reaction. 
Table I. Characteristics of the study population used for immunohistochemical staining.

\begin{tabular}{lcccc}
\hline Characteristic & S1 stage & S2 stage & S3 stage & S4 stage \\
\hline Age (years) & 21 & 24 & 32 & 38 \\
Sex & Male & Female & Male & Female \\
Etiology & HBV infection & HBV infection & HBV infection & HBV infection \\
Treatment & No & No & No & No \\
\hline
\end{tabular}

$\mathrm{HBV}$, hepatitis B virus.

Table II. Characteristics of the study population used for western blot analysis $(n=8)$.

\begin{tabular}{lccc}
\hline Characteristic & Total $(\mathrm{n}=8)$ & Periphery of carcinoma $(\mathrm{n}=5)$ & Hepatic fibrosis $(\mathrm{n}=3)$ \\
\hline Age (years) & $39.38 \pm 17.51$ & $50.6 \pm 9.79$ & $20.67 \pm 6.43$ \\
Sex (male:female) & $7: 1$ & $4: 1$ & $3: 0$ \\
Etiology & HBV infection & HBV infection & HBV infection \\
Degree of fibrosis & - & - & S4 \\
\hline
\end{tabular}

HBV, hepatitis B virus.

cytes in the control rats. Conversely, the number of hepatocytes positive for Arkadia staining was reduced in the liver samples from rats with hepatic fibrosis (Fig. 4A). Compared with in the control group, the mRNA expression levels of Arkadia were significantly increased in the fibrotic liver samples from rats in the $\mathrm{CCl}_{4} 6$ and 8 week groups $(\mathrm{P}<0.01$; Fig. 4B). Furthermore, western blot analysis revealed that the protein expression levels of Arkadia in the liver samples from the rats in the $\mathrm{CCl}_{4}$ 6 and 8 week groups were significantly reduced compared with in the control group $(\mathrm{P}<0.05$; Fig. $4 \mathrm{C}$ and $\mathrm{D})$.

Expression of Arkadia in patients with hepatic fibrosis. To examine the expression of Arkadia in the fibrotic livers of human patients, the remnants of human liver tissues were collected from biopsies conducted for routine clinical purposes, and fibrotic liver samples were collected during surgery. The characteristics of the study population are presented in Tables I and II. According to the region and extent of collagen deposition, and the severity of liver structural damage, patients were classified into stages S1-S4 of hepatic fibrosis (Fig. 5A). Hepatocyte swelling, cytoplasm rarefaction, spotty necrosis in the hepatic lobule and inflammatory cell infiltration into the portal duct areas were detected in the liver sample from the patient with S1 stage hepatic fibrosis. At S2 stage, numerous inflammatory cells had infiltrated into the portal areas, and a few fibrous septa formed by collagen fibers were located in the portal area; however, the structure of the liver lobule retained normal architecture. The liver lobules at S3 stage were disordered, alongside deposition of fibrous septa, inflammatory cell infiltration and hepatocyte degeneration. Eventually, pseudolobules were formed in the livers at S4 stage, and hepatic fibrosis progressed to cirrhosis.

Further immunohistochemical staining indicated that Arkadia was predominantly expressed in the cytoplasm, whereas expression was reduced in the nucleus of hepatocytes and cholangiocytes in the livers of human patients with various stages of fibrosis (Fig. 5A). There was no marked difference in the positive staining rate among the groups of patients with different stages of hepatic fibrosis (S1-S4 stages). Western blot analysis revealed that the protein expression levels of Arkadia were significantly lower in the fibrotic liver samples compared with samples from the periphery of hepatocellular carcinoma tissues $(\mathrm{P}<0.05$; Fig. 5B and $\mathrm{C})$. The samples from the periphery of hepatocellular carcinoma were considered the control.

\section{Discussion}

The present study investigated Arkadia expression in the livers of rats with hepatic fibrosis. The results indicated that Arkadia was highly expressed in the cytoplasm, whereas it was weakly expressed in the nucleus of cholangiocytes and hepatocytes from rat liver samples. Furthermore, reduced levels of Arkadia mRNA transcripts, but increased protein expression levels of Arkadia were detected in the livers of control rats. The mRNA expression levels of Arkadia were increased in the livers of rats with BDL-induced hepatic fibrosis, particularly at 7 days post-surgery. Subsequently, the mRNA expression levels of Arkadia were gradually reduced; however, they remained significantly higher compared with in the sham group, with the exception of the insignificant difference between the BDL 28 day group and sham group. Conversely, the relative protein expression levels of Arkadia were significantly reduced in the liver samples of rats with BDL-induced hepatic fibrosis compared with in the sham group; the levels were gradually reduced in the livers of rats with hepatic fibrosis throughout the observation period. In the $\mathrm{CCl}_{4}$-induced rat model of hepatic fibrosis, gradually increased levels of Arkadia mRNA transcripts and decreased levels of protein expression were detected in the livers of the $\mathrm{CCl}_{4}$-treated groups of rats. Similarly, significantly reduced levels of Arkadia protein were detected in the liver samples from patients with various stages of hepatic 

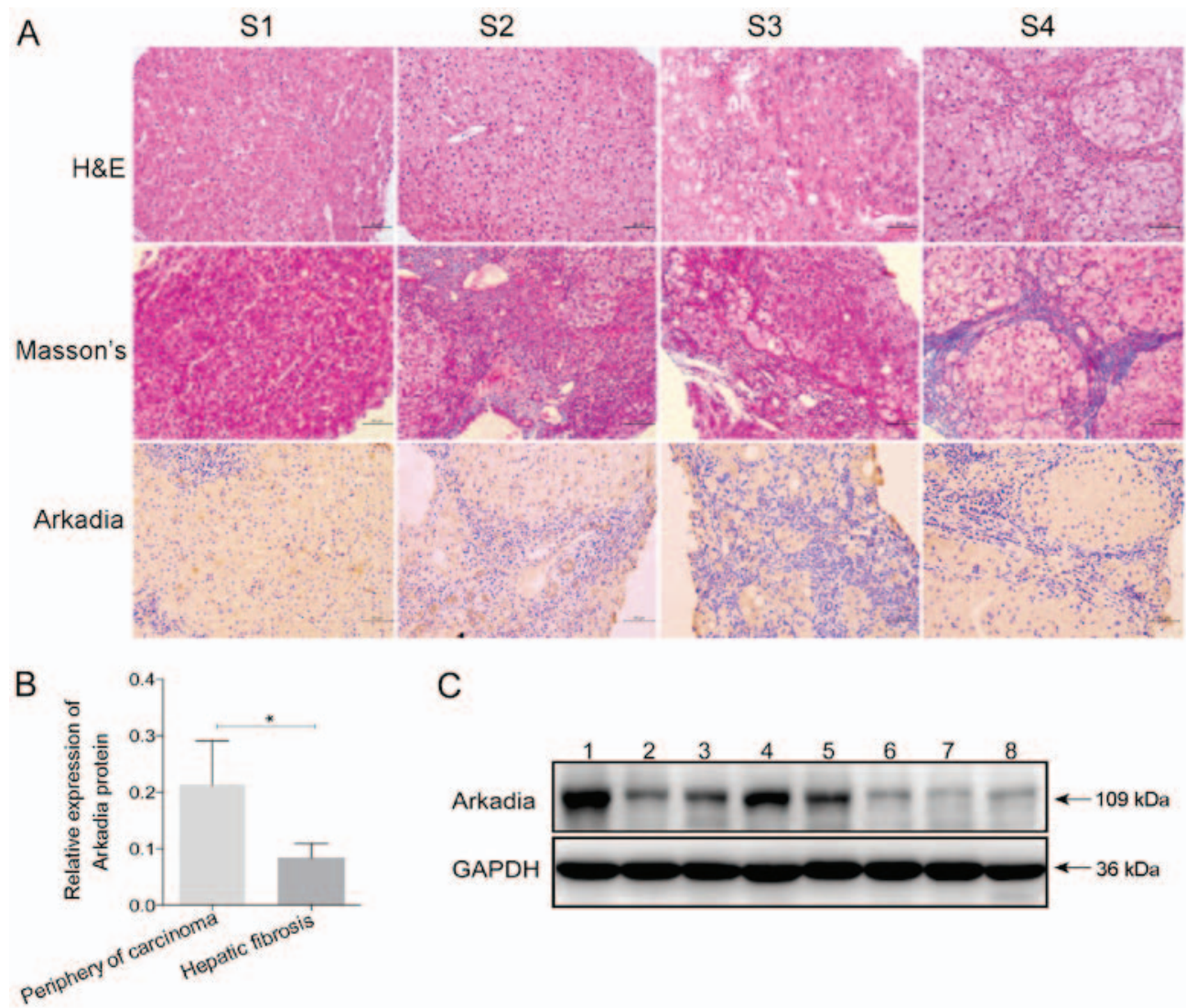

Figure 5. Arkadia expression in fibrotic liver samples from human patients. Liver samples were obtained from patients with hepatic fibrosis at various stages, and underwent H\&E and Masson staining. The protein expression levels of Arkadia were detected in fibrotic and nonfibrotic liver samples by immunohistochemistry and western blot analysis. Results are presented as representative images or are expressed as the means \pm standard deviation of individual groups. (A) Histological and immunohistochemical examination of liver sections (original magnification, x200). S1, S2, S3 and S4, hepatic fibrosis at the indicated stage. (B) Relative protein expression levels of Arkadia. (C) Western blot analysis of the protein expression levels of Arkadia. Lanes 1-5, periphery of carcinoma; lanes 6-8, hepatic fibrosis. ${ }^{*} \mathrm{P}<0.05$. H\&E, hematoxylin and eosin.

fibrosis. These findings suggested that Arkadia mRNA and protein expression may be inversely associated in fibrotic livers during the development and progression of hepatic fibrosis.

There are numerous potential reasons regarding the absence of a positive correlation between the mRNA and protein expression levels of Arkadia, and these may not be mutually exclusive $(19,20)$. Firstly, there are numerous complex and varied post-transcriptional mechanisms that regulate the translation of mRNA into protein, which are not yet sufficiently well defined to be able to compute protein concentrations from mRNA transcripts. A previous study reported that some genes exhibited minimal variation in their mRNA expression throughout the cell cycle and were likely to have little or no correlation with final protein level; the cells may control the open reading frames at the translational and/or post-translational level, with the mRNA levels being somewhat independent of final protein concentration (19). Furthermore, while a positive correlation does exist between mRNA and protein abundance, mRNA abundance is not a predictor of protein abundance in multicellular organisms. Secondly, proteins may differ in their in vivo half lives, as a result of varied protein synthesis and degradation (20). Cells can control the rates of degradation and synthesis for specific proteins, and there is significant heterogeneity, even within proteins that have similar functions $(21,22)$. Alternatively, it is possible that the ubiquitin proteasome system may degrade the Arkadia protein in the progression of hepatic fibrosis. We aim to further investigate how hepatic fibrosis regulates Arkadia expression in the liver.

In conclusion, these findings suggested that decreased Arkadia protein expression may be associated with the progression of hepatic fibrosis. Although several studies have reported that Arkadia positively contributes to renal tubular epithelial to mesenchymal transition through the degradation of Smad7 (23), the mechanistic roles of Arkadia are rarely reported in hepatic fibrosis. Therefore, the current findings will encourage us to further investigate how Arkadia affects the progression of hepatic fibrosis.

The present study hypothesized that Arkadia may participate in hepatic fibrosis. Using two hepatic fibrosis models induced by $\mathrm{BDL}$ and $\mathrm{CCl}_{4}$ intoxication, and human liver samples from patient with hepatic fibrosis, the results identified the expression and localization of Arkadia in hepatic fibrosis. The results indicated that Arkadia protein expression was significantly decreased, whereas the levels of Arkadia mRNA transcripts were elevated in fibrotic livers.

\section{Acknowledgements}

The present study was supported by the National Natural Science Foundation of China (grant no. 81300334). 


\section{References}

1. Puche JE, Saiman Y and Friedman SL: Hepatic stellate cells and liver fibrosis. Compr Physiol 3: 1473-1492, 2013.

2. Brenner DA, Waterboer T, Choi SK, Lindquist JN, Stefanovic B, Burchardt E, Yamauchi M, Gillan A and Rippe RA: New aspects of hepatic fibrosis. J Hepatol 32 (Suppl): 32-38, 2000.

3. Moreira RK: Hepatic stellate cells and liver fibrosis. Arch Pathol Lab Med 131: 1728-1734, 2007.

4. Lim YS and Kim WR: The global impact of hepatic fibrosis and end-stage liver disease. Clin Liver Dis 12: 733-746, 2008.

5. Richburg JH, Myers JL and Bratton SB: The role of E3 ligases in the ubiquitin-dependent regulation of spermatogenesis. Semin Cell Dev Biol 30: 27-35, 2014.

6. Nagano Y, Mavrakis KJ, Lee KL, Fujii T, Koinuma D, Sase H, Yuki K, Isogaya K, Saitoh M, Imamura T, et al: Arkadia induces degradation of SnoN and c-Ski to enhance transforming growth factor-beta signaling. J Biol Chem 282: 20492-20501, 2007.

7. Mavrakis KJ, Andrew RL, Lee KL, Petropoulou C, Dixon JE, Navaratnam N, Norris DP and Episkopou V: Arkadia enhances Nodal/TGF-beta signaling by coupling phospho-Smad2/3 activity and turnover. PLoS Biol 5: e67, 2007.

8. Ling H, Roux E, Hempel D, Tao J, Smith M, Lonning S, Zuk A, Arbeeny $\mathrm{C}$ and Ledbetter S: Transforming growth factor $\beta$ neutralization ameliorates pre-existing hepatic fibrosis and reduces cholangiocarcinoma in thioacetamide-treated rats. PLoS One 8: e54499, 2013

9. Massagué $\mathrm{J}$ and Wotton D: Transcriptional control by the TGF-beta/ Smad signaling system. EMBO J 19: 1745-1754, 2000.

10. Xu F, Liu C, Zhou D and Zhang L: TGF- $3 /$ SMAD pathway and its regulation in hepatic fibrosis. J Histochem Cytochem 64: 157-167, 2016.

11. Koinuma D, Shinozaki M, Komuro A, Goto K, Saitoh M, Hanyu A, Ebina M, Nukiwa T, Miyazawa K, Imamura and Miyazono K: Arkadia amplifies TGF-beta superfamily signalling through degradation of Smad7. EMBO J 22: 6458-6470, 2003.

12. Levy L, Howell M, Das D, Harkin S, Episkopou V and Hill CS: Arkadia activates Smad3/Smad4-dependent transcription by triggering signal-induced SnoN degradation. Mol Cell Biol 27 : 6068-6083, 2007

13. Le Scolan E, Zhu Q, Wang L, Bandyopadhyay A, Javelaud D, Mauviel A, Sun L and Luo K: Transforming growth factor-beta suppresses the ability of Ski to inhibit tumor metastasis by inducing its degradation. Cancer Res 68: 3277-3285, 2008.
14. Tsubakihara Y, Hikita A, Yamamoto S, Matsushita S, Matsushita N, Oshima Y, Miyazawa K and Imamura T: Arkadia enhances BMP signalling through ubiquitylation and degradation of Smad6. J Biochem 158: 61-71, 2015.

15. Aghaei I, Shabani M, Doustar N, Nazeri M and Dehpour A: Peroxisome proliferator-activated receptor- $\gamma$ activation attenuates motor and cognition impairments induced by bile duct ligation in a rat model of hepatic cirrhosis. Pharmacol Biochem Behav 120: 133-139, 2014.

16. Javadi-Paydar M, Ghiassy B, Ebadian S, Rahimi N, Norouzi A and Dehpour AR: Nitric oxide mediates the beneficial effect of chronic naltrexone on cholestasis-induced memory impairment in male rats. Behav Pharmacol 24: 195-206, 2013.

17. Uchinami H, Seki E, Brenner DA and D'Armiento J: Loss of MMP 13 attenuates murine hepatic injury and fibrosis during cholestasis. Hepatology 44: 420-429, 2006.

18. Livak KJ and Schmittgen TD: Analysis of relative gene expression data using real-time quantitative PCR and the 2(-Delta Delta C(T)) Method. Methods 25: 402-408, 2001

19. Greenbaum D, Colangelo C, Williams K and Gerstein M: Comparing protein abundance and mRNA expression levels on a genomic scale. Genome Biol 4: 117, 2003.

20. Khositseth S, Pisitkun T, Slentz DH, Wang G, Hoffert JD, Knepper MA and Yu MJ: Quantitative protein and mRNA profiling shows selective post-transcriptional control of protein expression by vasopressin in kidney cells. Mol Cell Proteomics 10: M110.004036, 2011.

21. Lian Z, Kluger Y, Greenbaum DS, Tuck D, Gerstein M, Berliner N, Weissman SM and Newburger PE: Genomic and proteomic analysis of the myeloid differentiation program: Global analysis of gene expression during induced differentiation in the MPRO cell line. Blood 100: 3209-3220, 2002.

22. Gerner C, Vejda S, Gelbmann D, Bayer E, Gotzmann J, Schulte-Hermann R and Mikulits W: Concomitant determination of absolute values of cellular protein amounts, synthesis rates, and turnover rates by quantitative proteome profiling. Mol Cell Proteomics 1: 528-537, 2002.

23. Liu FY, Li XZ, Peng YM, Liu H and Liu YH: Arkadia regulates TGF-beta signaling during renal tubular epithelial to mesenchymal cell transition. Kidney Int 73: 588-594, 2008.

This work is licensed under a Creative Commons Attribution-NonCommercial-NoDerivatives 4.0 International (CC BY-NC-ND 4.0) License. 